Proc. Indian Acad. Sci. (Eaqth Planet, Sci ${ }_{2}$, Vol, 90, No, 2, July 1981, pp. 141-146.

- Printed in India,

\title{
On the formation of a sharp layer of metallic constituents at sunrise in the lower thermosphere
}

\author{
J S SHIRKE*, R SRIDHARAN, S P GUPTA, \\ A D DANILOV + , A A POKHUNKOV ${ }^{+}, \mathrm{V} K$ SEMENOV + \\ and $V$ A VERFOLOMEEVt \\ Physical Research Laboratory, Ahmedabad 380 009, India \\ +Institute of Applied Geophysics Moscow, USSR
}

MS received 14 August 1980; revised 22 April 1981

\begin{abstract}
Results are presented of two instrumented rocket experiments per. formed from an equatorial station, one at night and the other shortly after sunrise. The ion neutral composition as well as electron density and the amplitude of plasma irregularities were monitored. During the latter flight, a sharp layer of ionisation with its lower boundary at $100 \mathrm{~km}$ was observed. The layer had a half width close to $1 \mathrm{~km}$ and a peak electron density of $5.6 \times$ $10^{4} \mathrm{~cm}^{-3}$. Large amplitude of plasma irregularities, noticed on the negative gradient portion of the layer indicates a downward direction of the polarisation electric field during the observations. The resulting downward drift of photoions as they are produced at sunrise followed by the local decrease of the drift is suggested to be the cause of the layer formation at that altitude. The long lasting nature of such layers once identified on ionograms indicates that they are constituted of metallic ions possibly of micrometeoritic origin deposited overnight in the lower thermosphere. The required photoionisation rate of production of the metallic ions at sunris eis about $2 \mathrm{~cm}^{-9} \mathrm{sec}^{-1}$.
\end{abstract}

Keywords. Ionospheric layer; photoionisation of metals; micrometeoritic flux; metallic layer; thermosphere.

\section{Introduction}

The presence of metallic ions, often in the form of discrete layers has been detected in the altitude region of $85-130 \mathrm{~km}$ through mass spectrometric measurements by several investigators (Istomin 1963, Narcisi and Bailey 1965; Young et al 1967; Narcisi 1968; Anderson and Barth 1971; Johannessen and Krankowsky 1972; Aikin and Goldberg 1973; Zbinden et al 1975). In the region noted above, $\mathrm{Fe}^{+}$and $\mathrm{Mg}^{+}$have been the most common ions even though $\mathrm{Si}^{+}$, $\mathrm{Na}^{+}, \mathrm{Ca}^{+}, \mathrm{K}^{+}$and other trace elements have also been detected. It is generally accepted that the metal ions are of meteoritic origin. The main processes of ionisation of the metallic atoms are through collisions with the ambient atmosphere, charge exchange with ambient molecular species and photoionisation. The production of ionisation through the collisional processes is maximum around $105 \mathrm{~km}$ (Lebedinets and Shuskova 1970a). This process is expected to take place throughout the day. The other two processes are likely to

\footnotetext{
*deceased
} 
be important in the lower thermosphere at sunrise particularly so the photoionisation, if neutral particles exist in large numbers, as is believed to be the case (Shirke and Sridharan 1979). The present experiment was planned to measure the rate of photoionisation of the metallic constituents at sunrise in the lower thermosphere. It was soon discovered that the ions accrete on formation to constitute a sharp layer. The characteristics of the layer are presented and the mechanism of its formation discussed.

\section{Instrumentation}

The experiments were performed on two Indian made Centaure rockets ISRO C 05.50 and C 05.51 on 21 December 1978. The launchings were made from Thumba, the equatorial rocket-launching site (dip lat. $0 \cdot 85^{\circ} \mathrm{S}$ ). One of the rockets 0550 was launched at 0230 and another at 0615 IST. The solar zenith angles corresponding to these flights were $134^{\circ}$ and $94^{\circ}$ respectively. The experiments formed part of a collaborative programme between the Depart ment of Space, India and the USSR State Committee on Meteorology. Atmospheric ion and neutral compositions and electron density were monitored on each of the rockets. The results of the composition studies of the major species have already been published (Danilov et al 1980). In this paper we present the results associated mainly with the metallic constituents.

The atmospheric composition was measured using a Bennett radio frequency mass speztrometer of tyre $M \times 6407$. The mass range of the instrument was 1.48 amu. The instrument was switched between the ion mode and neutral mode alternately every 1.4 seconds.

The electron density measurements were made using a standardized Langmuir probe (Satya Prakash and Subbaraya 1967). The sensor was well insulated from the rocket body by using a guard ring. As a result the frequency response of the instrument was adequate to measure the amplitude of electron density fluctuations with scale sizes as low as one meter in the vertical direction as well as the ambient electron density.

\section{Presentation of data}

In figure 1 is shown the electron density profile obtained in the upleg part of the night flight 05.50. The amplitude of irregularities in the electron density of scale sizes ranging between 1-15 meters is also shown as a function of altitude. An average electron density of the order of $3 \times 10^{3} \mathrm{~cm}^{-3}$ is sean in the altitude region 90 to $105 \mathrm{~km}$. A valley in the electron density profile is noticed between 120 to $140 \mathrm{~km}$. Large amplitude fluctuations are seen wi thin the valley region. Whether these are real or of instrumental origin is not very clear and this aspect is not considered further.

In figure 2 is shown the electron density profile obtained during the early morning flight (upleg). The main feature observed is an overall increase in electron density at all levels as a result of sunrise; the E-region sunrise time being 0550 IST. A very sharp layer of ionisation around $100 \mathrm{~km}$ is also observed. The valley which was present at night appears to have been filled up 


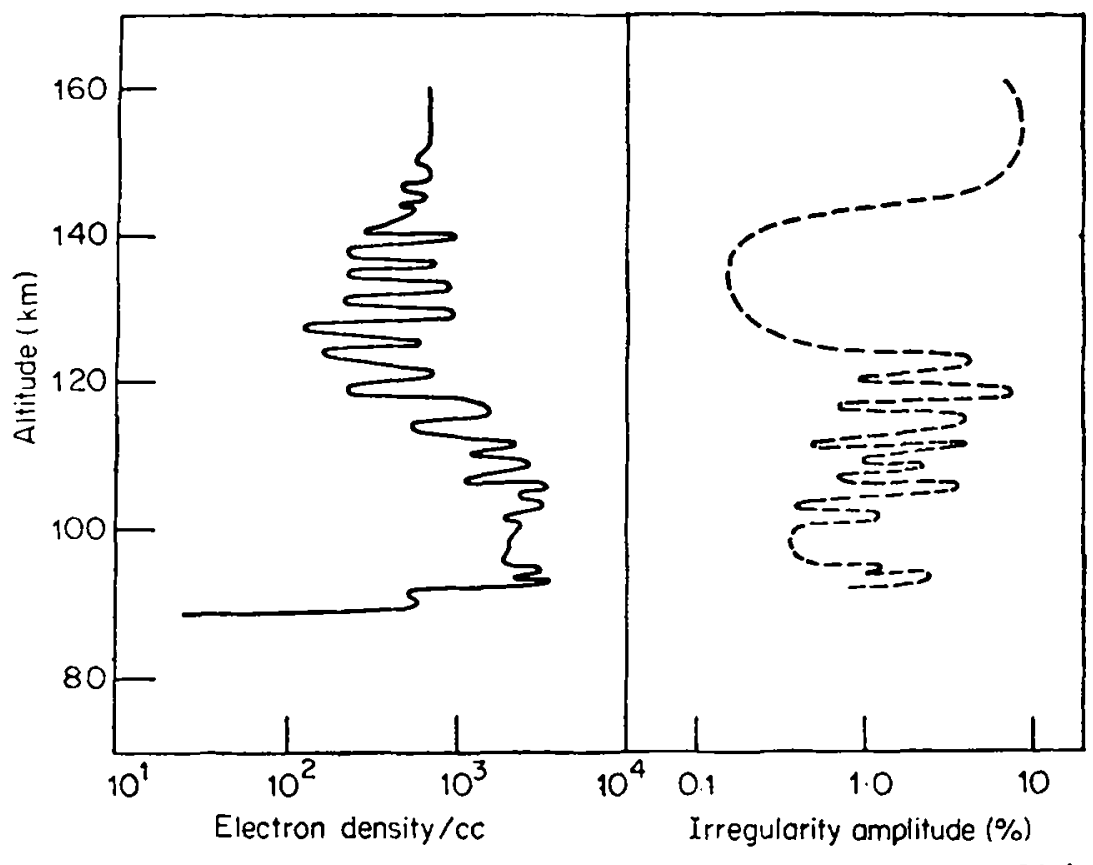

(scole: 1-15M)

Figure 1. Electron density profile made at Thumba at 0230 IST on 21 December 1978. Amplitude of irregularities in the $1-15 \mathrm{~m}$ scale sizes is also shown.



Figure 2. Flectron density profile made at 0615 IST from Thumba on 21 December 1978. Amplitude of irregularities in the $1-15 \mathrm{~m}$ scale sizes is also shown.

though partially by fresh ionisation produced following sunrise. Formation of fresh ionisation in the neighbourhood of $75 \mathrm{~km}$ is also noticed. The overall features of both upleg and downleg data were the same in both the flights, and therefore only the upleg part is reproduced. The sharp layer was observed 
exactly at the same altitude with the same peak electron density of $5.6 \times 10^{4} \mathrm{~cm}^{-3}$, in the early morning flight. Further in the electron density profiles reproduced, variations less than 200 meters were smoothed out manually.

The amplitude of irregularities in the 1 to $15 \mathrm{~m}$ scale sizes was also measured. The amplitude seems to have been enhanced substantially around $101 \mathrm{~km}$. This can be attributed to the 'gradient instability' mechanism (Satya Prakash et al 1969). The irregularities above $130 \mathrm{~km}$ were of vehicular origin and therefore are not discussed. It was shown (Reid 1968) that 'gradient type' plasma irregularities are generated when the vertical electric field and plasma density gradient are in the same sense. In the present case, the existence of large amplitudes of irregularities on the topside of the layer, that is, on the negative gradient portion, confirms that the polarization electric field was downward and the electron/ion drift must have continued to be downward during the measurements, as is normally the case throughout the night.

The electron density corresponding to the peak of the sublayer at $100 \mathrm{~km}$ is seen in figure 2 to be $5.6 \times 10^{4}$ electrons $\mathrm{cm}^{-3}$ while the ambient density is $1.4 \times 10^{4}$ electons $\mathrm{cm}^{-3}$. The half width of the layer is close to $1 \mathrm{~km}$ and the columnar electron density within the layer is $4.5 \times 10^{9}$ electrons $\mathrm{cm}^{-2}$ over and above the ambient level.

The results of the ion composition measurements in the morning flight have revealed $\mathrm{NO}^{+}$as the main positive ion in the neighbourhood of the sublayer with a density of $1.4 \times 10^{4} \mathrm{~cm}^{-3}$ (Danilov et al 1980). The night flight also has revealed $\mathrm{NO}^{+}$as the main ion component throughout the lower thermosphere. In addition Sitions constituted about $10 \%$ of the ambient density.

Returning to the results of the early morning flight, the ion composition within the layer could not be monitored because of the short transit time of the rocket within the layer compared to the sweep rate of the mass spectrometer. However, it is reported (Shirke and Sridharan 1979) that once formed such layers last for several hours. For example in the equatorial region their existence can be monitored on ionograms until they reach the peak of the $F$ region due to upward drift during day time. This shows that the ions associated with the layers must be metallic in origin for which the recombination coefficient is known to be about five orders lower than for the molecular species like $\mathrm{NO}^{+}$and $\mathrm{O}_{2}{ }^{+}$(Swider 1969).

Ground-based ionosonde measurements made on the day of the rocket launchings and on the succeeding day have shown the initiation of group retardation effects on the $h^{\prime} f$ trace between the interval 0547 and 0553 IST. This marks the start of production of ionisation in the lower thermosphere around 25 minutes prior to the launching of the second rocket. The vertical drift of ionisation typically lies between 10 to $20 \mathrm{~m} \mathrm{sec}^{-1}$ for an equatorial station (Woodman 1970). Assuming a mean downward drift of $15 \mathrm{msec}^{-1}$ within 25 minutes prior to the launching, the freshly produced ionisation would move down by $23 \mathrm{~km}$.

\section{Discussion}

The main question which arises is to how a layer as sharp as has been observed could be produced in the lower thermosphere. Direct production of 
ionisation in a limited region is the first thing which comes to mind. However, this would require the layer to be formed in just a few tens of seconds and in an altitude region of a few hundred meters if it is not to be widened by the prevailing downward drift of ionisation. Such an extremely selective process is hard to conceive. Furthermore the accumulation of neutral atom is even more difficult.

The mechanism which has received much attention in literature for layer formation is that of wind shear. Such a mechanism has been favoured for explaining the density stratifications during evening hours when low recombination coefficient exists (Ananda Rao et al 1977). In the present case however the time required for the observed accretion of ions in the layer works out to be several orders larger than what is available. Wind shear has therefore to be ruled out as the main agency for the layer formation.

One of the most plausible mechanism for the formation of the sharp layer seems to be a large decrease (or even reversal) of the drift below $100 \mathrm{~km}$. Under normal circumstances the field and therefore the vertical drift decrease with a scale height of about $6 \mathrm{~km}$ below $100 \mathrm{~km}$. But at sunrise when a transition in the field and in the drift direction occur the situation seems to be different. Balsley and Woodman (1969) have shown that in the morning the vertical drift in the $F$-region has smooth transiton from the night time downward to the daytime upward direction. In the $E$-region however they have shown that the east-west drift suddenly reduces from a large eastward velocity $(600 \mathrm{~m} / \mathrm{sec})$ to near zero and remains so for a long time. This period varies from day to day, but generally exceeds 30 minutes. However, it is well-known that elestrojet radar drift measurements are not accurate when the drift velocity is smaller than about $50 \mathrm{~m} / \mathrm{sec}$. Therefore the above radar measurements cannot be taken as a roof that the $E$-region drifts remain equal to zero for a long time.

The possibility of wind generated local electric fields also as a mechanism could not be ruled out. The wind generated electric fields at $100 \mathrm{~km}$ and below are known to be able to affect equatorial electric fields by resulting in a large decrease (or even reversal) of the drift at these altitudes (Prakash and Pandey 1979). Such a large decrease in the fields would eventually result in the formation of a sharp layer.

Regarding the origin of metallic ions themselves, collisional ionisation of meteoritic particles is a normal source in the lower thermosphere. However, this process is not confined to the sunrise period alone. Furthermore the maximum rate of production of ions through this process is $10^{-1} \mathrm{~cm}^{-3} \mathrm{sec}^{-1}$ around $105 \mathrm{~km}$ (Lebedinets and Shuskova 1970a). On the other hand it is also shown that maximum amount of sputtering of micrometeoritic dust occurs around $120 \mathrm{~km}$ (Lebedinets and Shuskova 1970b). An overnight accumulation of the micrometeoritic dust can be an ideal source of metallic atoms for the layer formation.

If an average downward drift of $15 \mathrm{msec}^{-1}$ were to be assumed for micrometeoritic ions then half the ionisation within a column of $23 \mathrm{~km}$ would accumulate at $100 \mathrm{~km}$ within 25 minutes of the time elapsed between the initiation of photoionisation and the time of measurements. If the entire columnar 
density of $4.5 \times 10^{9}$ ions $\mathrm{cm}^{-2}$ within the layer over and above the ambient density level were presumed to be metallic, then their production rate close to $2 \mathrm{~cm}^{-3} \mathrm{sec}^{-1}$ is required to explain the observations.

It may by noted that the meteor activity is lowest annually in February and highest during the period June to August (Hawkins 1956). The Geminid $\mathrm{m}$ siower takes place between 7 to 15 December and the Ursid shower between 17 and 24 December. The rocket experiment was conducted on 21 December. The meteor activity on this day is found to represent the mean astivity for the year. The calculated rate of photoionisation of meteoritic particles could therefore be taken as corresponding to the mean yearly activity.

\section{Conclusion}

The rate of photoionisation of metallic atoms for a short period at sunrise is shown to be around 20 times larger than the normal rate of production due to ablation of meteorites. A plausible mechanism is shown by which accretion of these metallic ions, as they are formed, can take place in the morning. Such layers once formed are long lasting and have great importance in the gereration of plasma irregularities and sporadic $E$ layers.

\section{References}

Aikin A C and Goldberg R A 1973 J. Geophys. Res. 78734

Ananda Rao, Raghavendra Rao B R and Reddy C R 1977 J. Geophys. Res. 821510

Anderson J G and Barth C A 1971 J Geophys. Res. 763723

Balsley B B and Woodman R F 1969 J. Atmos. Terr. Phys. 31865

Danilov A D, Pokhunkov A A. Semenov V K, Vorfolomeev V A, Shirke J S, Sridharan RC

Gupta S P and Pradhan S N 1980 Adv. Space Exploration Vol. 7 Low latitude Aeronomi, Processes P. 111

Hawkins G S 1956 Mon. Not. R. Astron. Soc. 116103

Istomin V G 1963 Space Res. 3209

Johannessen A and Krankowsky D 1972 J. Geophys. Res. 772888

Lebedinets V N and Shushkova V B 1970 Planet. Space Sci. 18 1653, 1659

Narcisi R S and Bailey A D 1965 J. Geophys. Res. 703687

Narcisi R S 1968 Space Res. 8360

Prakash S and Pandey R 1979 Proc. Indian Acad. Sci. (Earth Planet. Sci.) 88229

Reid G C 1968 J. Geophys. Res. 731627

Satya Prakash, Gupta S P and Subbaraya B H 1969 Rad. Sci. 4791

Satya Prakash and Subbaraya B H 1967 Rev. Sci. Instrum. 381132

Shirke J S and Sridharan R 1979 Proc. Indian Acad. Sci. (Earth Planet. Sci.) 88219

Swider W Jr 1909 Planet. Space Sci. 171233

Woodman R F 1979 J. Geophys. Res. 756249

Young J M, Johnson C Y and Holmes J C 1967 J. Geophys. Res. 721473

Zbinden P A, Hidalgo M A, Eberhardt P and Geiss J 1975 Planet. Space Sci. 231621 